\title{
NANOPLEX: A REVIEW OF NANOTECHNOLOGY APPROACH FOR SOLUBILITY AND DISSOLUTION RATE ENHANCEMENT
}

\author{
POONAM MADHUKAR KASAR*, KALYANI SUNDARRAO KALE, DIPTI GANESH PHADTARE
}

\author{
R. G. S Institute of Pharmacy, Anjaneri, Nashik, Maharashtra, India \\ Email: sonikasar2706@gmail.com
}

Received: 20 Apr 2018, Revised and Accepted: 10 Jun 2018

\begin{abstract}
The use of nanotechnology based on the development and fabrication of nanostructures is one approach that has been employed to overcome the challenges involved with conventional drug delivery systems. Formulating Nanoplex is the new trend in nanotechnology. Nanoplex means drug nanoparticle complex with the oppositely charged polyelectrolyte. In this technique, the cationic or anionic drug is reacted with an oppositely charged polyelectrolyte to form a complex. Compared with other nanostructures, the yield of Nanoplex is greater and the complexation efficiency is better. The nanoplex formed shows improved solubility and dissolution rate compared to raw drug crystals. Nanoplex formulation is characterized through the production yield, complexation efficiency, drug loading, particle size and zeta potential using scanning electron microscopy, differential scanning calorimetry, X-ray diffraction and dialysis studies. Nanoplex have wide-ranging applications in different fields such as cancer therapy, gene-drug delivery, drug delivery to the brain and protein and peptide drug delivery.
\end{abstract}

Keywords: Nanotechnology, Nanoplex, Polyelectrolyte, Solubility enhancement, Dissolution

(C) 2018 The Authors. Published by Innovare Academic Sciences Pvt Ltd. This is an open access article under the CC BY license (http://creativecommons.org/licenses/by/4.0/] DOI: http://dx.doi.org/10.22159/ijcpr.2018v10i4.28467

\section{INTRODUCTION}

Nanotechnology is the science that deals with the nano length scale and with processes that occur at the molecular level. There are a number of examples from nature of structures with nano dimensions such as red blood corpuscles (RBCs), viruses, DNA and water molecules [1]. Even our history has a number of examples that prove we have exploited the advantages of technology in one or another form. The term 'nanotechnology' has also been used in other fields of science such as electronics, physics and engineering for many decades. Nanotechnology has led to tremendous progress in these fields. Nanotechnology is a multidisciplinary field, representing a convergence of the basic sciences and applied disciplines such as biophysics, molecular biology, and bioengineering [2]. It has had a powerful impact in various fields of medicine, including cardiology, immunology, ophthalmology, oncology, endocrinology and Pneumology, and in highly specialized areas such as gene delivery, brain targeting and oral vaccine formulation. Nanotechnology provides intelligent materials, systems and devices for better pharmaceutical applications [3-5]. Nanoparticles are defined as particulate dispersions or solid particles with a size in of the range 10-100 $\mathrm{nm}$. Drugs are dissolved, entrapped, encapsulated or attached to nanoparticle matrices [6, 7].

\section{Types of nanostructures}

\section{Nanosuspensions}

A nanosuspension is defined as a very finely colloid, biphasic, dispersed, solid drug particle in an aqueous vehicle, of size below 1 $\mu \mathrm{m}$, without any matrix material, stabilized by a surfactant and a polymer, prepared using a suitable method for drug delivery applications through various routes of administration such as the oral, topical, parenteral, ocular and pulmonary routes [8-10].

\section{Solid lipid nanoparticles}

Solid lipid nanoparticles are novel potential colloidal carrier systems used as alternative materials to polymers. They are identical to oilin-water emulsions used for parenteral nutrition, but the liquid lipid of these emulsions is replaced by a solid lipid. They have good compatibility and low toxicity and are physically stable. Lipophilic drugs are better delivered using solid lipid nanoparticles $[11,12]$.

\begin{abstract}
Nanofibres
Nanofibres are defined as fibres with diameter less than 50-500 nm. They have an extremely high surface area-to-weight ratio compared with conventional nonwovens. Nanofibres have good filtration properties $[13,14]$.
\end{abstract}

\section{Nanocomposites}

Nanocomposites have superior strength and fracture toughness. Nanocomposites have good mechanical, optical, electrical and thermal properties [15].

\section{Carbon nanotubes}

Carbon nanotubes (CNTs) are allotropes of carbon. The length-todiameter ratio of these nanostructures is greater than $1,000,000$. CNTs exhibit good carrier properties and are used to transport biomolecules to target sites. These biomolecules include a diverse array of compounds such as drugs, vaccines, small peptides, proteins, nucleic acids, vitamins and sugars [16-18].

\section{Nanopores}

Materials with pore-sizes in the nanometre range are of special interest for a broad range of industrial application because of their outstanding properties with regard to thermal insulation and controllable material separation and release and their suitability as templates or fillers for chemistry and catalysis. Aerogels are examples of nonporous materials. They are produced using sol-gel chemistry [19].

\section{Liposomes}

Liposomes are small artificial vesicles (50-100 nm) developed from phospholipids. They have been used as drug carriers as a result of their ability to prevent degradation of drugs, reduce side effects and target drugs to sites of action. Liposomes are used for transdermal drug delivery (they enhance the skin permeation of drugs of high molecular weight and poor water solubility) and as carriers for delivery of drugs (they reduce toxicity) in the treatment of ocular diseases [20].

\section{Polymeric nanoparticles}

Polymeric nanoparticles are solid colloidal particles with a diameter 
ranging from $1 \mathrm{~nm}$ to $1000 \mathrm{~nm}$. Polymeric, nanoparticulate systems developed using biodegradable and biocompatible polymers offer interesting options for controlled drug delivery and drug targeting [21].

\section{Polymeric micelles}

Polymeric micelles are self-assembled core-shell nanostructures formed in aqueous solutions consisting of amphiphilic block polymers. Micelles are formed in aqueous solutions when the concentration of the block copolymer increases above a certain concentration or critical micelle concentration [22].

\section{Dendrimers}

Dendrimers are branched macromolecules. They have a central core unit with a high degree of molecular uniformity, a narrow molecular weight distribution, specific size and shape characteristics and a highly functionalized, terminal surface. The cellular uptake in dendrimers is through endocytosis, and drugs are bound to dendrimers within cells [23-25].

\section{Nanocapsules}

Nanocapsules are vesicular or reservoir systems in which oil/water is essentially confined to a cavity surrounded by a tiny polymeric membrane. Nanocapsules can be targeted to specific cells and locations within the body after they are injected intravenously or subcutaneously [26].

\section{Nanoplex}

A nanoplex is a drug nanoparticle complex with an oppositely charged polyelectrolyte. Cationic or anionic drugs are made to reach with oppositely charged polyelectrolytes to form Nanoplex [27].

\section{Magnetic nanoparticles}

Magnetic nanoparticles form a class of engineered particulate materials of particle size less than $100 \mathrm{~nm}$ that can be manipulated using external magnetic fields. Magnetic nanoparticles consist of a core of a polymer such as a dextran coated with an inorganic substance such as iron oxide [28]

\section{Nanoemulsions}

Nanoemulsions/submicron emulsions are thermodynamically stable, transparent (translucent) dispersions of oil and water stabilized interfacial films of surfactant and cosurfactant molecules. Their droplet size is less than $100 \mathrm{~nm}$ [28].

\section{Quantum dots}

Quantum dots are semiconducting materials consisting of a semiconductor core provided with a shell to improve optical properties. Their properties originate from their physical size, with the radius being in the range 10-100 $\mathrm{A}$. These have had a significant impact on imaging, in vitro and in vivo detection and analysis of biomolecules, immunoassay and DNA hybridization. They are used in non-viral vectors for gene therapy. Their main application is in labelling cells and as therapeutic tools for cancer treatment [29].

\section{Carbon allotrope graphenes}

Graphene is a substance made of pure carbon, with atoms arranged in a regular hexagonal pattern similar to that of graphite but in a one-atom-thick sheet. It is an allotrope of carbon whose structure is a single planar sheet of sp2-bonded carbon atoms that are densely packed in a honeycomb crystal lattice. Hence, graphenes may be considered as the mother of graphite, fullerene and CNTs [30].

\section{Preparation of amorphous drug Nanoplex}

Depending on the solubility (which is a function of $\mathrm{pH}$ ), a drug is dissolved in an acidic or basic aqueous solution to form it cation or anion, respectively. Amphoteric drugs forms cations when they are dissolved in aqueous acetic acid solutions (AA) $(\mathrm{pH}<\mathrm{pKa})$, whereas acidic drugs form anions when they are dissolved in aqueous $\mathrm{KOH}$ solutions $(\mathrm{pH}>\mathrm{pKa})$. The ionized drug solution is subsequently added to the oppositely charged aqueous polyelectrolyte solution, upon which a drug-polyelectrolyte electrostatic interaction takes place to form a soluble drug-polyelectrolyte complex, as illustrated in fig. 1.

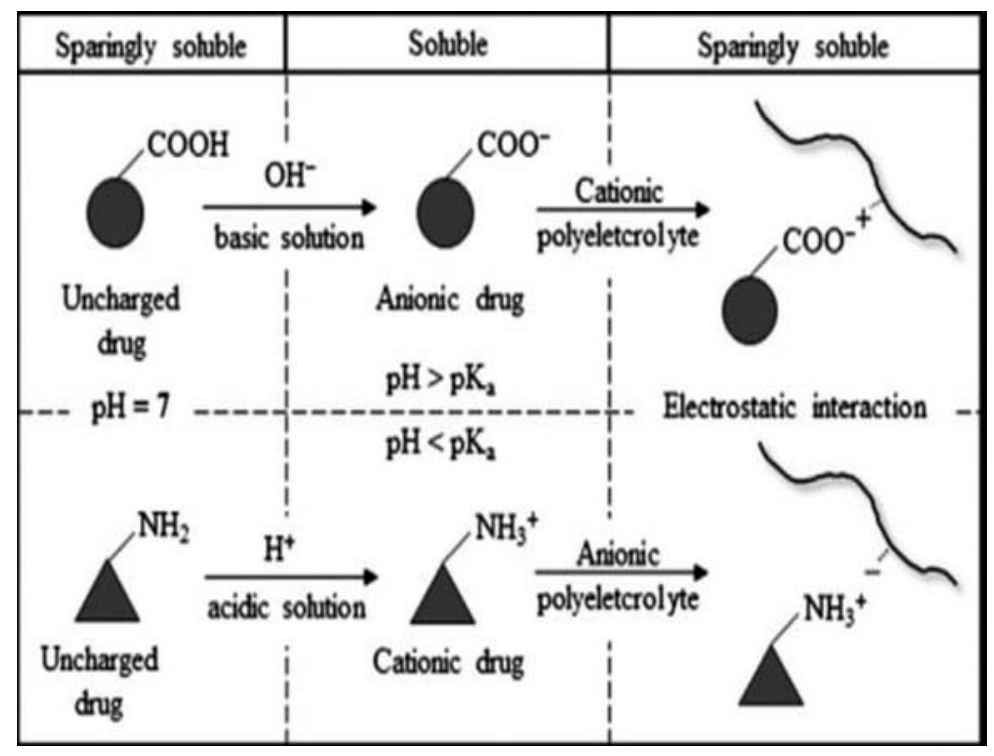

Fig. 1: Preparation of nanoplex

At a certain concentration, the value of which depends on the drug hydrophobicity, drug-PE complexes aggregate as a result of the inter drug hydrophobic interaction and finally form an insoluble drug nanoplex in the presence of a salt. The role of the salt is to provide a charge screening effect to minimize repulsions of the like-charged PE chains, which can inhibit complex aggregation, as shown in fig. 1. The strong drug-PE electrostatic interaction prevents the drug molecules from assembling into ordered crystalline structures upon precipitation, resulting in the amorphous nanoplex [31].

\section{Mechanism of nanoplex formation}

The water-insoluble drug is first dissolved in an acidic or basic medium to form an anionic or cationic drug solute. Afterwards, the ionized drug solution is mixed with an oppositely charged polyelectrolyte solution, which initiates the drug-polyelectrolyte electrostatic interaction and simultaneously charges neutralization as 
shown in fig. 2. The drug solute is transformed back to its sparingly soluble form upon charge neutralization, leading to a loss of solubility, and hence there is rapid precipitation and formation of the nanoscale drug-polyelectrolyte complex. The combination of rapid precipitation and strong electrostatic interactions between the drug and the polyelectrolyte prevents the drug molecules from assembling into ordered crystalline structures. As a result, an amorphous drugpolyelectrolyte nanoparticle complex is formed [32].

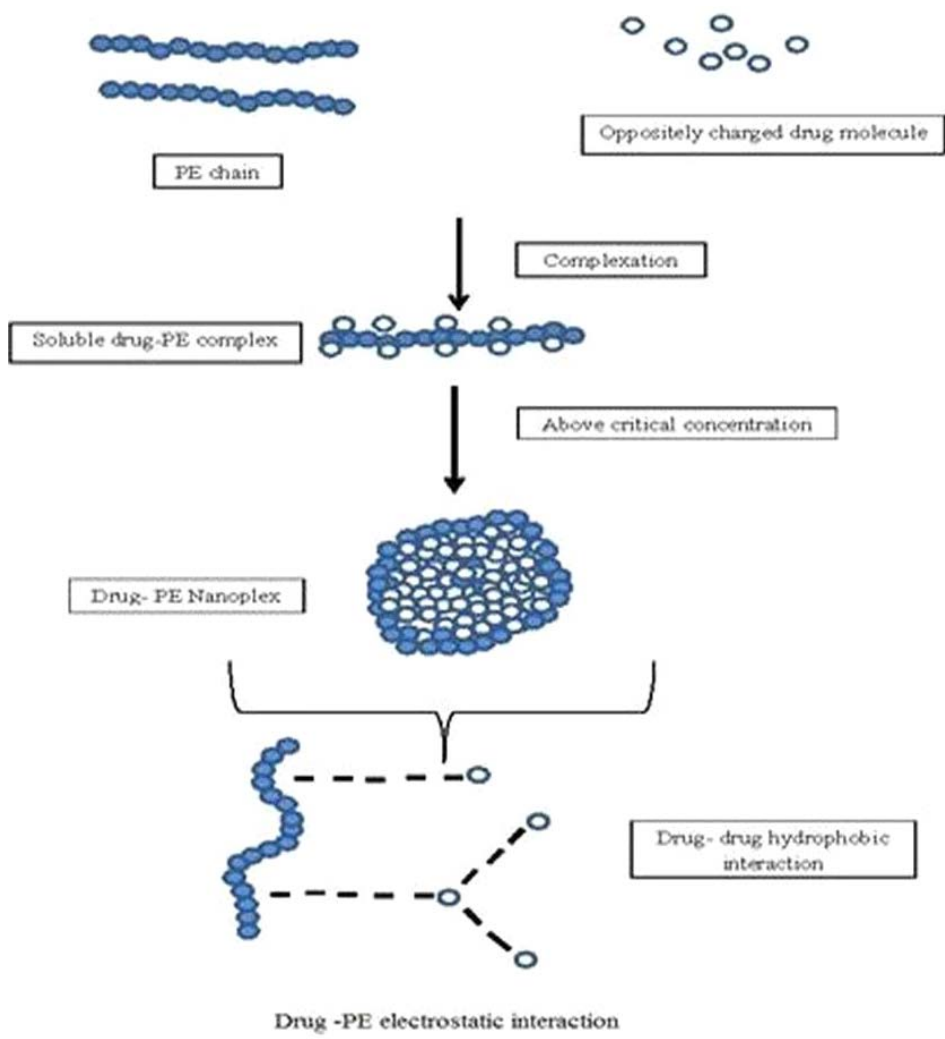

Fig. 2: Mechanism of nanoplex formulation

\section{Advantage of nanoplex}

1. The method of preparation of Nanoplex is simple as the only mixing of two solutions, of the drug and PE, is involved.

2. Nanoplex preparation does not require a heavy use of solvents.

3. The energy requirement for nanoplex preparation is minimal compared with other nanoformulations.

4. Nanoplex formation is a fast process.

5. There is no need to use sophisticated instruments to prepare Nanoplex.

\section{Role of salt in nanoplex preparation}

The salt plays an important role in the self-assembly of the nanoplex through its electrostatic charge shielding function. In the absence of salt, intermolecular repulsions between the like-charged PE chains inhibit their spatial interactions. The same can be said for the drug, with the charged drug molecules repelling if there is no salt, thus hindering a compact assembly of drug molecules. The higher solubility of the drug in a solution of lower ionic strength further contributes to the lack of inter-drug hydrophobic interactions. [33]Consequently, there is no nanoplex precipitation in the absence of a salt despite the oppositely charged interactions. Instead, soluble drug-PE complexes may be present, reminiscent of the soluble amphiphilic drug and PEs previously investigated. In the, presence of a salt, the shielding of charge enables the PE chains to be in closer contact with each, other. Likewise, the drug molecules are also in closer contact, which leads to the formation of a, core-shell nanoplex, which then precipitates upon drug. Nevertheless, the charge-shielding effect of,the salt must not be too strong, or it will (i) inhibit the electrostatic interactions between the drug molecules and PE involved in the complex formation or (ii) neutralize the charge interactions between the drug and PE in the already-formed complex, resulting in decomplexation [34].

\section{Evaluation of nanoplex}

- Complexation efficiency: This is defined as the mass of a drug that forms a complex with the polyelectrolyte relative to the initially added drug. It is calculated by measuring the optical density of the supernatant after the first centrifugation of the nanoplex suspension [31].

- Production yield: It is the ratio of the weight of the drug and polyelectrolyte initially added to the weight of the dry nanoplex formed after freeze-drying [31].

- Drug loading: This is the actual amount of drug present in the nanoplex powder. It is calculated by dissolving $5 \mathrm{mg}$ of the nanoplex powder in $20 \mathrm{ml}$ of ethanol and measuring the absorbance of the solution after centrifugation and filtration [31].

- Particle size analysis: The particle size of the nanoplex is analysed using a particle size analyser [31].

- Zeta potential: The zeta potential of the nanoplex is determined using a zetasizer [35].

- Differential scanning calorimetry (DSC): DSC is performed to determine the interactions between the drug and polyelectrolyte. The interactions are also determined on the basis of their melting points, and the difference is calculated using a DSC instrument. The results are presented in the form of graphs [31].

- Powder X-ray diffraction: Powder X-ray diffraction patterns of samples are determining using a powder X-ray diffractometer. 
This gives an idea about the nature of the sample [35].

- Scanning electron microscopy: The surface morphology of the sample is observed using a scanning electron microscope [31].

- Dissolution study: Dissolution testing of the nanoplex is carried out using the dialysis bag method to determine the drug release [31].
- Saturation solubility study: The solubility of the drug and that of the formulation are determined using the orbital flask shaker method. The concentration of the drug is determined from the absorbance, through spectrophotometric analysis [31].

- Stability study: The stability of the nanoplex is tested by placing it in an environmental stability chamber, and its drug content is determined [31].

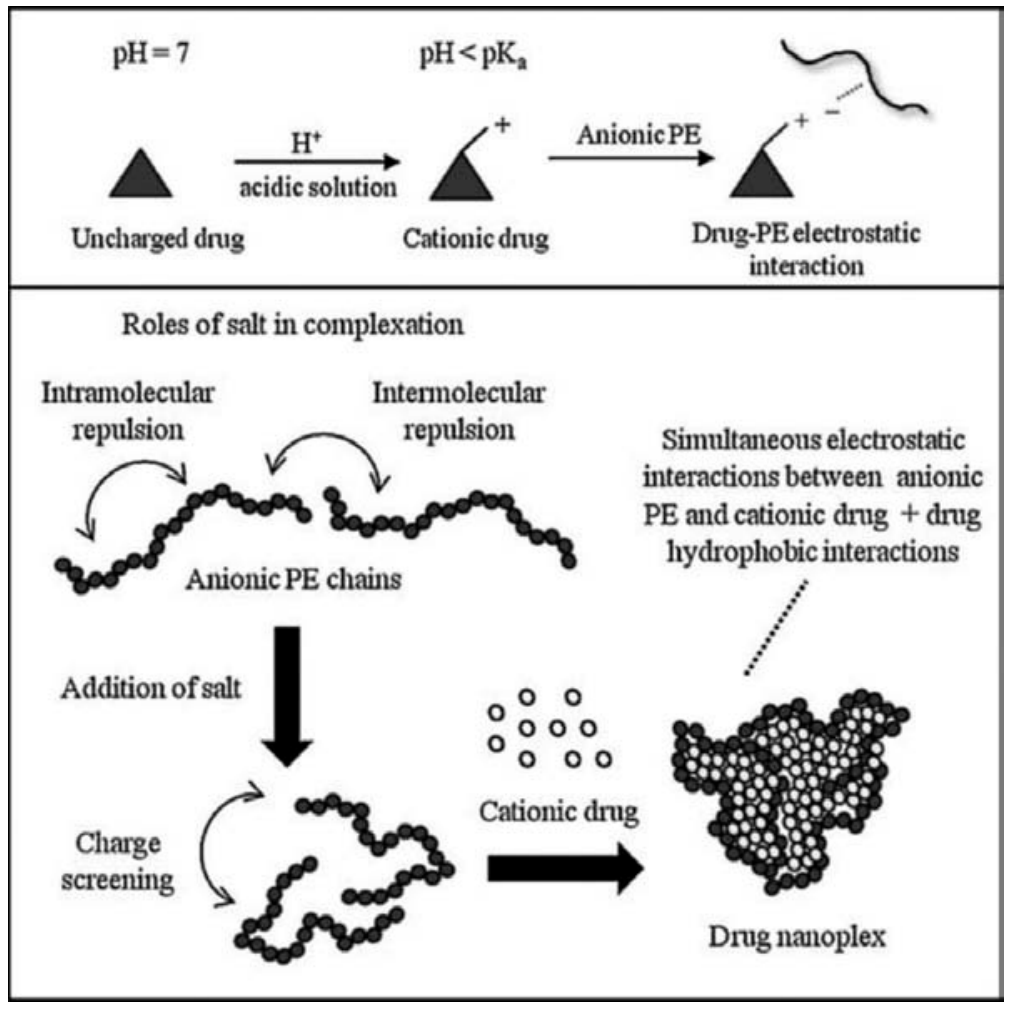

Fig. 3: Role of salt in nanoplex formulation

\section{Applications of nanoplex}

\section{Enhancement of solubility and dissolution rate}

Preparing Nanoplex of poorly water-soluble drugs (i.e., class II and class IV drugs) enhances their solubility and dissolution rate and ultimately bioavailability. Solubility enhancement reduces the dose requirement of a drug [31].

\section{Drug delivery to the brain}

The blood-brain barrier (BBB) is the most important factor limiting the development of a new drug for the central nervous system. The BBB is characterized by relatively impermeable endothelial cells with tight junctions, enzymatic activity and active efflux transport systems. It effectively prevents the passage of water-soluble molecules from the blood circulation into the CNS and can reduce the concentration of lipid-soluble molecules in the brain through the action of enzymes or efflux pumps. Consequently, the BBB only permits selective transport of those molecules that are essential for brain function. It has been suggested that nanoparticles may be used as non-viral gene delivery vectors and that they have great potential in therapeutic applications for several diseases such as HIV-1, AIDS, dementia and cerebral ischaemia. Nanoplex have been evaluated for the specificity and efficiency of quantum dot (QD) complexes [with MMP-9-siRNA (nanoplex) in downregulating the expression of the MMP-9 gene in the brain microvascular endothelial cells (BMVECs) that constitute the BBB. Adela Bonoiu et al. demonstrated the use of a novelnanoplex siRNA delivery system in modulating MMP-9 activity in BMVECs and other MMP-9-producing cells. This application will prevent neuroinflammation and maintain the integrity of the BBB [36]

\section{Nanoplex for gene delivery}

Non-viral vector-mediated gene therapy is currently one of the most attractive strategies used due to their biocompatibility, biodegradability, minimal cytotoxicity, lack of pathogenicity and low immunogenicity. The development of non-viral vectors consisting of dendrimers, liposomes and cationic polymers is the focus of research currently. Cationic polymers have been found to be useful in neutralizing the anionic nature of DNA, thus efficiently condensing DNA and allowing it to enter cells easily. Cationic polymers represent an interesting class of vectors in plasmid DNA delivery formulations [37].

\section{Drug targeting in cancer treatment}

Many different cationic lipids have been synthesized for non-viral vectors. The general structure of a cationic lipid has three parts: (1) a hydrophobic lipid anchor group, which helps form the micellar structure and can interact with cell membranes; (2) a linker group, such as an ester, an amido group or a carbamate; and (3) a positively charged head-group, mainly composed of cationic amines. Cationic cholesterol derivatives could be selected as the anchor group because of their high transfection activity and low toxicity. The linker group of cationic cholesterol derivatives controls the conformational flexibility, degree of stability, biodegradability and gene transfection efficiency. Cationic nanoparticles composed of $\mathrm{OH}-$ Chol (NP-OH) could deliver siRNA with a high transfection efficiency in vitro when the nanoparticle/siRNA complex (nanoplex) is formed in an $\mathrm{NaCl}$ solution [38]. 


\section{Drug delivery of proteins and peptides}

Significant advances in biotechnology and biochemistry have led to the discovery of a large number of bioactive molecules and vaccines based on peptides and proteins. The development of suitable carriers remains a challenge because the epithelial barrier of the gastrointestinal tract limits the bioavailability of these molecules and they are susceptible to gastrointestinal degradation by digestive enzymes. Proteins are charged molecule that forms a complex with polyelectrolyte therefore, they are a suitable candidate for nanoplex formulation $[39,40]$

\section{CONCLUSION}

By preparing nanoplex of poorly water-soluble drug we can increase its solubility and hence dissolution rate. Most orally administered drugs are amphiphilic in nature and soluble in a weak acid or base. They can be transformed into amorphous Nanoplex by the simple complexation method. The preparation of a nanoplex requires only the mixing of two solutions at ambient conditions. It is also solventfree and fast. It requires little energy and produces uniform-sized nanoparticles with a high complexation efficiency, drug loading and production yield.

\section{AUTHORS CONTRIBUTIONS}

All the author have contributed equally.

\section{CONFLICT OF INTERESTS}

Declared none

\section{REFERENCES}

1. Ochekpe NA, Olorunfemi PO, Ngwuluka NC. Nanotechnology and drug delivery. part 1: nanostructures for drug delivery. Trop J Pharm Res 2009a;8:265-74.

2. Manivannan R. Nanotechnology: a review. J Appl Pharm Sci 2011;1:8-16.

3. Bhattacharyya D, Singh S, Satnalika N, Khandelwal A, Jeon S. Nanotechnology, big things from a tiny world: a review. Int J U E-Ser Sci Technol 2009;2:29-38.

4. Malakar J, Ghosh A, Basu A, Nayak A. Nanotechnology: a promising carrier for intracellular drug delivery system. Int Res J Pharm 2012;3:36-40.

5. Lamba D. A brief review on nanotechnology. Impulse 2006;2:58-63.

6. Mohanraj VJ, Chen Y. Nanoparticles-a review. Trop J Pharm Res 2006;5:561-73.

7. Manmode AS, Sakarkar DM, Mahajan NM. Nanoparticles tremendous therapeutic potential: a review. Int J Pharm Tech Res 2009;1:1020-9.

8. Nagare SK, Ghughure SM, Salunke SB, Jadhav SG, Dhore RJ. A review on: nanosuspension an innovative acceptable approach in the novel delivery system. Uni J Pharm 2012;1:19-31.

9. Prabhakar $\mathrm{CH}$, Balakrishna K. A review on nanosuspension in drug delivery. Int J Pharma Bio Sci 2011;2:549-58.

10. Verma A, Bindal MC. Nanosuspension: advantages and disadvantages. Ind J Nov Drug Delivery 2012;4:179-88.

11. Ekambaram P, Sathali A, Priyanka K. Solid lipid nanoparticle: a review. Sci Rev Chem Commun 2012;2:80-102.

12. Pragati S, Kuldeep S, Ashok S, Satheesh M. Solid lipid nanoparticle: a promising drug delivery technology. Int J Pharm Sci Nanotech 2009;2:509-16.

13. Kattamuri SB, Potti L, Vinukonda A, Bandi V, Changantipati S, Mogili RK. Nanofibers in pharmaceuticals-a review. Am J Pharmtech Res 2012;2:187-212.

14. Dineshkumar B, Krishnakumar K, Bhatt AR, John A, Paul D, Cherian J, et al. Nanofibers: potential applications in wound care management. Adv Poly Sci-Tech 2012;2:30-2.

15. Sambarkar PP, Patwekar SL, Dudhgaonkar BM. Polymer nanocomposites: an overview. Int J Pharm Pharma Sci 2012;4:60-5.

16. Hirlekar R, Yamagar M, Garse H, Mohit VIJ, Kadam V. Carbon nanotubes and its application: a review. Asian J Pharm Clin Res 2009;2:17-27.

17. Mishra R, Mishra A. Review on potential applications of carbon nanotubes and nanofibers. Int J Pharm Rev Res 2013;3:12-7.
18. Gurjar PN, Chouksey S, Patil G, Naik N, Agrawal S. Carbon nanotubes: pharmaceutical applications. Asian J Biomed Pharm Sci 2013;3:8-13.

19. Wanunu M. Nanopores: a journey towards dna sequencing. Phys Life Rev 2012;9:125-58.

20. Ochekpe NA, Olorunfemi PO, Ngwuluka NC. Nanotechnology and drug delivery. Part 2: Nanostructures for drug delivery. Trop J Pharm Res 2009b;8:275-87.

21. Jawahar N, Meyyanathan SN. Polymeric nanoparticles for drug delivery and targeting: a comprehensive review. Int J Health Allied Sci 2012;1:217-23.

22. $\mathrm{Xu} \mathrm{W}$, Ling $\mathrm{P}$, Zhang T. Polymeric micelles, a promising drug delivery system to enhance bioavailability of poorly watersoluble drugs. J Drug Delivery 2013;1-15. http://dx.doi.org/ 10.1155/2013/340315.

23. Mishra IN. Dendrimer: a novel drug delivery system. J Drug Delivery Ther 2011;1:70-4.

24. Shishu G, Maheshwari M. Dendrimers: the novel pharmaceutical drug carriers. Int J Pharm Sci Nanotech 2009;2:493-502.

25. Trivedi V, Bhimani B, Patel U, Daslaniya D, Patel G, Vyas B. Dendrimer: polymer of 211st century. Int J Pharm Res Biosci 2012;1:1-21.

26. Dineshkumar B, Krishnakumar K, John A, Paul D, Cherian J, Panayappan L. Nanocapsules: a novel nano-drug delivery system. Int J Res Drug Delivery 2013;3:14-6.

27. Cheow WS, Hadinoto K. Self-assembled amorphous drugpolyelectrolyte nanoparticle complex with enhanced dissolution rate and saturation solubility. J Colloid Interface Sci 2012;367:518-26.

28. Indira TK, Lakshmi PK. Magnetic nanoparticle-a review. Int J Pharm Sci Nanotechnol 2010;3:1035-42.

29. Patel RP, Joshi JR. An overview on nanoemulsion: a novel approach. Int J Pharm Sci Res 2012;3:4640-50.

30. Bera D, Qian L, Tseng T, Holloway PH. Quantum dots and their multimodal applications: a review. Materials 2010;3:2260-345.

31. Cheoww S, Hadinoto K. Self-assembled amorphous drugpolyelectrolyte nanoparticle complex with enhanced dissolution rate and saturation solubility. J Colloid Interface Sci 2012c;367:518-26.

32. Cheow WS, Hadinoto K. Green preparation of antibiotic nanoparticle complex as potential anti-biofilm therapeutics via self-assembly amphiphile-polyelectrolyte complexation with dextran sulfate. Colloids Surf B Biointerfaces 2012b;92:55-63.

33. Singhs K, Caram-Lelham N. Thermodynamics of kappacarrageenan-amphiphilic drug interaction as influenced by specific counterions and temperature: a microcalorimetric and viscometric study. J Colloid Interface Sci 1998;203:430-46.

34. Hugerth A, Sandlot LO. The effect of polyelectrolyte counterion specificity, charge density, and confirmation on polyelectrolyteamphiphile interaction: the carrageenan/furcellaranamitriptyline system. Biopolymers 2001;58:186-94.

35. Cheow WS, Hadinoto K. Green amorphous nanoplex as a new supersaturating drug delivery system. Langmuir 2012a;28:6265-75.

36. Bonoiu A, Mahajan SD, Ling Y, Ding H, Ken-Tye Yong, Nair B, et al. MMP-9 gene silencing by a quantum dot-sirna nanoplex delivery to maintain the integrity of the blood-brain barrier. Brain Res 2009;1282:142-55.

37. Thomas JJ, Rekha MR, Sharma CP. Dextran-protamine polycation: an efficient nonviral and haemocompatible gene delivery system. Colloids Surf B 2010b;81:195-205.

38. Hattori Y, Hagiwara A, Ding W, Maitani Y. Nacl improves sirna delivery mediated by nanoparticles of hydroxyethylated cationic cholesterol with amido-linker. Bioorg Med Chem Lett 2008;18:5228-32.

39. Woitiski C, Ribeiro A, Neufeld R. Bioencapsulation into nanoplex carrier for oral insulin delivery. In: International Workshop On Bioencapsulation. Vienna Au Annals 2007;15:720-44, 4-13.

40. Ranjan A, Pothayee N, Seleem M, Jain N, Sriranganathan N, Riffle JS, et al. Drug delivery using novel nanoplex against a salmonella mouse infection model. J Nanopart Res 2010;12:905-14. 